\title{
LA EDUCACIÓN A LO LARGo DE LA VIDA, PRIORIDAD DE LA UNIÓN EUROPEA
}

\author{
$\mathrm{M}^{\mathrm{a}}$ Luisa Sarrate Capdevila \\ $U N E D$
}

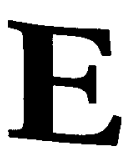

I interés de la Unión Europea por la educación ha sido tardío. Ha habido que esperar a la década de los setenta para que se produzca una clara preocupación al respecto puesta de manifiesto, principalmente, en materia de formación profesional. En los últimos años, sin embargo, se viene observando un importante cambio, pues se está prestando especial atención a los temas educativos. Todo parece señalar que los países miembros han tomado conciencia de la necesidad de fomentar la creación de una identidad europea, sustentada en valores culturales para lograr una auténtica y duradera unidad. Paralelamente, las instituciones comunitarias están efectuando una decidida apuesta por la educación a lo largo de la vida, expresión que se equipara a la de educación permanente, y vienen subrayando la necesidad de impulsarla, a fin de promover no sólo el progreso económico, sino también el perfeccionamiento personal, la igualdad de oportunidades, la cohesión social y el desarrollo de la dimensión europea.

El objetivo de este trabajo radica en presentar los elementos básicos que han conformado y conforman en la actualidad la educación a lo largo de la vida en el marco de la Unión Europea. Con este propósito se ofrecen, en primer lugar, los principales rasgos que caracterizan el tratamiento otorgado a la educación desde el inicio de la actividad comunitaria, incidiendo en los planteamientos adoptados relativos al ámbito objeto de estudio, lo que permite constatar la evolución producida. En segundo lugar, se examinan los presu- 
puestos que han contribuido a impulsar esta nueva concepción educativa, en concreto, la relevancia otorgada en nuestra sociedad del conocimiento al capital inmaterial y que generó la proclamación de 1996 como Año Europeo de la Educación y Formación Permanentes. El conjunto de análisis realizados facilita la identificación de los criterios esenciales que configuran en nuestros días la política de la Unión en el ámbito objeto de estudio. Finalmente, se presentan los nuevos horizontes que se abren a la educación a lo largo de la vida en el marco de la entidad supranacional a la que pertenecemos.

\section{LA EDUCACIÓN Y LA UNIÓN EUROPEA}

Los criterios y políticas comunitarios en materia educativa no han sido tan homogéneos y coherentes como cabría esperar, por lo que resulta interesante analizar los principales elementos que configuran la transformación mantenida desde meros esquemas utilitarios, en los que la formación venía a concretarse en un instrumento para la capacitación profesional requerida, hasta planificaciones actuales, en las que la educación a lo largo de la vida alcanza un plano sustancial de objetivo comunitario, que merece la formulación de estrategias de desarrollo y la programación de realizaciones concretas. En este sentido, en el desarrollo de la cooperación comunitaria distinguimos las tres etapas siguientes: Los compromisos iniciales (1957-1979), Los Nuevos enfoques (1980-1989) y Los modernos Tratados y programas educativos (de 1990 a nuestros días).

La primera etapa se caracteriza, en líneas generales, por la tardanza en el tratamiento del campo educativo. Generalmente se afirma, no sin falta de razón, que la educación ha sido la gran ausente en el comienzo del proceso seguido para la construcción de la Unión Europea. No es de extrañar esta situación, puesto que ha de recordarse que el proyecto comunitario fue primero Comunidad Económica Europea, y luego Mercado Común, y si el nombre sigue siendo el signo y símbolo de una realidad, habrá que reconocer que el arranque original se encuentra marcado por un manifiesto interés por el sector económico (García Mínguez, 1998, p. 64), interés que sigue manteniéndose hoy día. De todas formas, ya en el Tratado de Roma de 1957 se incluyen tres artículos de merecida atención: El 57 atiende al reconocimiento mutuo de diplomas y títulos, el 118 contempla un apunte sobre el perfeccionamiento profesional y en el 128 se encomienda al Consejo que establezca los principios para la actuación común en formación profesional. Este marco legal pone en evidencia una especial preocupación por la capacitación de los recursos humanos, enfoque que va a prevalecer durante largo tiempo.

La lentitud de los primeros pasos se hace patente en el hecho de que hay que esperar hasta 1971 para que los Ministros de Educación se reunieran por vez primera y dictaminaran que la educación no debería considerarse como 
un simple elemento al servicio del progreso económico. Cuatro años más tarde se crea el Centro Europeo para el Desarrollo de la Formación Profesional (CEDEFOP) y, en 1976, se aprueba el Primer Programa de Acción en Educación, con el que puede decirse que comienza la Europa de la educación. Este primer programa identificó los seis ámbitos prioritarios que marcarán luego los principales campos de intervención; son los siguientes: Formación cultural y profesional de los ciudadanos de otros Estados miembros, mejora de la correspondencia entre los sistemas educativos, compilación documental y estadística, cooperación en materia de enseñanza superior, refuerzo del aprendizaje de lenguas extranjeras y desarrollo de la igualdad de oportunidades educativas, procurando especial atención a los jóvenes que abandonan el sistema escolar, a las mujeres y a la integración de los minusválidos.

Los hechos acontecidos muestran que los Estados miembros van tomando conciencia de la necesidad de procurar un fundamento cultural al proceso económico. En este periodo puede decirse que está presente, aunque de forma todavía muy incipiente, cierta preocupación por una educación a lo largo de la vida, al haberse abordado aspectos como la igualdad de oportunidades, la formación profesional continua, la lucha contra el analfabetismo y la educación de personas adultas.

En la segunda etapa, correspondiente a la década de los ochenta, se constata un nuevo enfoque de la cooperación educativa más coherente e integrador, que tiene por objeto responder a los nuevos problemas y demandas sociales generados por la crisis económica de finales de los setenta que afectó a gran parte del continente. Este nuevo enfoque, de acuerdo con Rodríguez, V.M (1993, p. 14), presenta dos efectos claros. Por una parte, se da prioridad a las medidas formativas para facilitar a los jóvenes la transición a la vida activa y, por otra, definitivamente se asume que la educación y la formación son dos realidades inseparables; por lo tanto sólo desde una perspectiva política que las integre se puede contribuir eficazmente al desarrollo social y económico del continente.

A mediados de esta década se intensifica la cooperación en enseñanza Superior, la incorporación del conocimiento de Europa en los currículos escolares, la integración de los minusválidos, y se marcan líneas de actuación concretas para la lucha contra el analfabetismo. Así mismo, por vez primera se hizo alusión a la gran importancia de la educación y la cultura en la construcción europea (Informe del Comité para la Europa de los ciudadanos, 1985). En 1986 se inicia el comúnmente denominado periodo de los programas, en que se aprueban y llevan a cabo un amplio número de ellos. Entre los más importantes cabe citar los siguientes: COMMETT (cooperación entre universidad y empresa), ERASMUS (intercambios universitarios), IRIS (formación de la mujer), PETRA (formación inicial y profesional de los jóvenes), HELIOS (integración de los minusválidos) y LINGUA (conocimiento de lenguas extranjeras). Todas estas actividades apenas tienen repercusión en el Acta Única Europea, firmada en 1986, donde el campo educativo queda relegado. 
En general, se constata que las instituciones comunitarias van asumiendo competencias algo más extensas y se detecta mayor voluntad política de acercamiento y de trabajo en común. Una de las conquistas más relevantes es haber logrado, no sin falta de esfuerzo, una interpretación amplia de los pocos artículos del Tratado de Roma relacionados con la educación, perspectiva sancionada por el Tribunal de Justicia (1985 y 1987), con ocasión de la aprobación de los programas COMMET y ERASMUS. Respecto al campo que nos ocupa, ya comienza a aludirse a la necesidad de introducir este principio en la acción educativa comunitaria al objeto de completar, continuar y ampliar los estudios iniciales de educación general y, especialmente, de formación profesional.

Los modernos Tratados y programas educativos de los años noventa constituyen los ejes principales en torno a los cuales gira la actividad educativa comunitaria en la tercera etapa que distinguimos. Es ésta una fase de reorganización y de ordenamiento integral en la que se logran avances significativos. Los primeros años son, en lo que respecta a las nuevas iniciativas, una continuación de los precedentes. Con todo, conviene indicar que aparecen nuevos programas como el FORCE, cuyo objetivo es impulsar la formación continua, y el EUROTECNET, centrado en la formación profesional y cambios tecnológicos. Igualmente, han de destacarse dos aspectos novedosos: La especial atención que recibe la enseñanza abierta y a distancia y la expansión de las relaciones internacionales al firmarse acuerdos de colaboración en enseñanza superior y formación profesional con países de Europa central y oriental y con Estados Unidos y Canadá.

El Tratado de la Unión Europea de Maastrich (1992) ratifica y eleva a categoría de legislación comunitaria la mayor parte de las acciones de cooperación educativa emprendidas anteriormente, consolidándose los principios de subsidiariedad, complementariedad y cooperación (Medina Rubio, 1999, pp. 8-11). Significa un considerable adelanto al situar en los artículos 126 y 127 las bases jurídicas de la política educativa relativas al desarrollo de una educación de calidad y de la política de formación profesional, respectivamente. Además, el artículo 3 del Título II establece los ámbitos de actuación comunitaria que tienen por objeto: Contribuir al desarrollo de una educación de calidad, potenciar la dimensión europea, instaurar una política de formación profesional y apoyar el florecimiento de la cultura de los Estados miembros.

Entre los años 1993 y 1994, la Comisión presentó nuevas propuestas para racionalizar y armonizar las acciones educativas comunitarias, reagrupándose a partir de 1995 en torno a tres macroprogramas: Sócrates, (destinado a fomentar una educación de calidad), Leonardo da Vinci (centrado en la formación profesional inicial y continua) y La Juventud con Europa (orientado a promover el desarrollo de intercambios y actividades entre los jóvenes para que participen en la construcción europea). Esta nueva generación de programas se distingue por incidir en la cooperación existente entre las tres grandes 
líneas de actuación expuestas y por tener como fin esencial facilitar e impulsar la educación a lo largo de la vida, planteamiento que se mantiene en la nueva edición que comprende los años 2000 a 2005.

Posteriormente, los artículos 149 y 150 del Tratado de Amsterdam (1997) dedicados a la educación reproducen, prácticamente, los de Maastrich; sin embargo, se observa cierto progreso, puesto que en su preámbulo se recoge de forma explícita la decisión de los Estados miembros de promover el desarrollo del nivel de conocimiento más elevado posible para los pueblos mediante un amplio acceso a la educación y su continua actualización (Comisión Europea, 1999, p. 95).

Como se puede advertir, en los planteamientos sobre educación efectuados por la Unión Europea ha prevalecido, en términos generales, un propósito esencial: El desarrollo económico, y un único camino: Favorecer la competitividad de los recursos humanos. Sin embargo, en esta etapa, gracias a las acciones emprendidas, especialmente a los programas educativos realizados, se detecta un acercamiento entre los sistemas educativos de los países europeos, generando una cultura de cooperación comunitaria y favoreciendo enfoques globales más próximos y no exclusivamente nacionales.

En lo que concierne a la educación a lo largo de la vida, se constata un giro importante al contemplarse explícitamente la necesidad de que el proceso educativo debe concebirse como una tarea global inacabada, al tiempo que emparejada a toda la trayectoria vital de la persona. Además, este principio educativo comienza a configurarse con una identidad propia en tanto que elemento esencial de la política educativa comunitaria, aspecto que se plantea con más detalle en las siguientes líneas.

\section{IMPORTANCIA DEL CAPITAL INMATERIAL EN LA SOCIEDAD COGNITIVA}

La creencia, ampliamente extendida, en el poder de mejora que posee la educación, como medio de reforma y progreso social, se constata al observar la creciente demanda de educación por parte de la población, la inclusión de la dimensión formativa en las medidas y acciones de índole social, así como en el hecho de que el nivel de recursos dedicados a la misma se considera como un índice del grado de desarrollo alcanzado por cada país.

De hecho, un presupuesto extensamente aceptado en nuestra época sostiene que la educación constituye un elemento clave dentro del complejo proceso de potenciar el crecimiento económico, de conseguir la modernización política y social y de formar ciudadanos con espíritu crítico, independientes 
y participativos. Fruto de la premisa de que la educación es uno de los impulsores del desarrollo, se la suele considerar como elemento sustancial en todo programa gubernamental y medida de imprescindible referencia al plantear cualquier actividad orientada a conseguir el progreso de la población. Esta creencia ha sido avalada por numerosos estudios, que ponen de manifiesto el hecho de que las naciones con un nivel elevado de logros educativos son las más avanzadas en las áreas de la industria, la ciencia, la tecnología, el bienestar social y la estabilidad política.

Una de las preocupaciones más relevantes de la Unión Europea reside en la promoción del desarrollo sostenible de las economías de su territorio; por ello no es de extrañar que se haya ocupado ampliamente de este tema, pues, además, presenta unos rasgos peculiares dadas las características de la sociedad del conocimiento en la que vivimos. Indudablemente, la relevancia que están adquiriendo las nuevas tecnologías de la información y de la comunicación resulta un fenómeno que está transformando el modelo de demanda de mano de obra cualificada a escala mundial, por lo que cada día cobra mayor incidencia la adquisición de conocimientos y habilidades de forma continua.

En nuestra sociedad, el conocimiento y la información, sin duda, están siendo los ejes fundamentales del ámbito de la producción y de la competitividad. Se está transformando el contexto de la actividad económica y la manera en que funciona la sociedad. En este marco, la sociedad europea está en transición hacia un nuevo modelo, en el que es imprescindible desarrollar habilidades para la ocupación y la vida económica. En concreto, en el ámbito económico, el capital humano, también denominado energía intelectual o, en terminología empleada por la Comisión Europea, capital inmaterial es hoy tan imprescindible como en otro tiempo lo fue la energía física; además las herramientas procesadoras de información y de conocimiento se equiparan hoy a lo que en tiempos de la revolución industrial significaron las máquinas.

\section{POLÍTICA SOCIOEDUCATIVA: FORMACIÓN DE RECURSOS HUMANOS}

La teoría del capital humano mantiene la tesis de que existe una relación directa entre el poder productivo de los trabajadores educados formalmente y el incremento del crecimiento económico. Este presupuesto se ha propagado con celeridad y ha obtenido gran popularidad que conserva, con altibajos, hasta nuestros días. De hecho, estudios recientes de diversas organizaciones internacionales subrayan la creciente influencia del cometido que desempeñan los conocimientos, las capacidades y las destrezas de los individuos, esto es, lo que conocemos como capital humano, en el crecimiento económico, en la prosperidad y también en el bienestar social. Por lo tanto la inversión en educación genera beneficios económicos y sociales, al permitir que se desa- 
rrolle en mayor medida el acervo de conocimientos y capacidades. Para ser efectiva esta inversión, como subraya la $\operatorname{OCDE}(1997$, p. 30), ha de ser equilibrada, esto es, debe afectar tanto a la escolaridad inicial y posterior como a promover la adquisición de conocimientos a lo largo de la vida.

Las reflexiones y propuestas para afrontar esta problemática otorgan un protagonismo esencial al mencionado capital inmaterial, expresión que engloba, según dictamina la Comisión Europea, en primer lugar la educación, además de las cualificaciones, la aptitud para la innovación y las tradiciones (Libro Blanco Crecimiento, Competitividad, Empleo. Retos y pistas para entrar en el siglo XXI, 1994). Desde estos planteamientos, la formación de los recursos humanos resulta un factor esencial en una sociedad que exige la recomposición y reconstrucción permanente del conocimiento.

La educación a lo largo de la vida se identifica como la estrategia más conveniente para responder al gran reto constituido por el triángulo del relanzamiento del crecimiento, la restauración de la competitividad y el restablecimiento de un nivel de empleo socialmente aceptable. En este sentido, una de las prioridades de las acciones a emprender, con el fin de poder hacer frente a la competencia internacional creando, al mismo tiempo, los puestos de trabajo necesarios, reside en apostar por la educación y la formación permanentes. No es éste un simple enunciado, sino que los responsables comunitarios ofrecen las implicaciones que conlleva el adoptar en la práctica dicha propuesta, al facilitar tanto las metas generales a conseguir como observaciones precisas relativas a las innovaciones que deben emprender los sistemas educativos para lograr saber y saber hacer en cualquier etapa de la vida. Entre dichas medidas, destacamos las siguientes:

- Aumentar las reservas de capital humano, eliminando el analfabetismo y la falta de competencias básicas de los jóvenes que abandonan la escuela, elevando el nivel de la formación profesional inicial e impulsando la inversión en formación continua.

- Efectuar innovaciones curriculares, lo que significa efectuar cambios en la naturaleza del contenido de las enseñanzas. Junto con las destrezas básicas se considera indispensable fomentar la aptitud para aprender, para comunicar, para trabajar en grupo, para analizar, diagnosticar y evaluar, así como el desarrollo de la autonomía, la independencia de espíritu y la capacidad de análisis basadas en el saber. En suma, se trata de generar posibilidades para mejorar la propia formación (saber y saber hacer) en cualquier momento de la vida.

- Establecer estrategias adecuadas como la formación en alternancia, los sistemas de rotación de puestos de trabajo, los créditos y permisos para la formación y las acciones de discriminación positiva para aquellos colectivos menos cualificados o que tienen mayores dificultades para incorporarse al mundo del trabajo. 
En suma, se trata de asentar las bases para institucionalizar el ejercicio de un auténtico derecho a la educación permanente, fundamentalmente, como instrumento de una política activa laboral, en el convencimiento de que la inversión en educación y formación genera claros beneficios económicos. Este presupuesto se llevó a la práctica, en gran medida, en la Cumbre de Luxemburgo (noviembre de 1997), en la que los Jefes de Gobierno y de Estado de los países de la Unión, al reconocer que la formación de los trabajadores constituye una herramienta indispensable para combatir el desempleo, asignaron sumas elevadas en los diversos planes quinquenales presentados para la capacitación de los recursos humanos como elemento esencial para el desarrollo de los pueblos.

\section{UN PARADIGMA EMERGENTE: LA SOCIEDAD DEL CONOCIMIENTO}

Junto a las anteriores observaciones, ha de tomarse en consideración que, en nuestros días, están ocurriendo una serie de transformaciones, como la explosión acelerada de la producción de conocimientos que conlleva un efecto de innovaciones importantes y cuya difusión discurre por los cauces de las telecomunicaciones, lo que está conformando un escenario totalmente nuevo para la información y comunicación humana. Paralelamente, el desarrollo tecnológico produce la necesidad de adquirir nuevos conocimientos y de renovarlos constantemente. Estos fenómenos, junto con otros, como la situación cambiante del trabajo, la creciente diversidad cultural y étnica, la problemática medio-ambiental y el envejecimiento de la población repercuten de forma decisiva en la educación y en la formación.

En efecto, la educación se encuentra ante la compleja situación de dar respuesta al advenimiento de la sociedad de la información, la civilización científico-técnica y la mundialización de la economía, según sostiene la Comisión en el Libro Blanco sobre la educación y la formación. Enseñar y aprender. Hacia la sociedad cognitiva (1995). Estos "tres choques motores" influyen en la evolución hacia una sociedad donde el conocimiento se presenta como elemento esencial, no sólo en el ámbito de la producción, sino también en el de las relaciones sociales y de la convivencia mundial.

En este nuevo escenario, de acuerdo con los responsables comunitarios, la educación a lo largo de la vida aparece como un elemento básico, presente en las propuestas ofrecidas para dar cumplida respuesta a los nuevos retos planteados, especialmente en las relativas a las necesidades de:

- Extender la cultura general. Esta función principal de la escuela ha de tener por objeto eliminar la marginación que provoca la ausencia de conocimientos fundamentales. Comporta la exigencia de una base cultural sólida y amplia, literaria y filosófica, científica, técnica y práctica que 
debe orientarse principalmente a desarrollar la autonomía e incitar a aprender a aprender a lo largo de toda la vida. Las transformaciones experimentadas obligan a fomentar la capacidad de captar el significado de las cosas, de interpretar y seleccionar y de desarrollar el juicio crítico.

- Promover aptitudes para el empleo y la actividad. Los conocimientos básicos, los técnicos, la creatividad y la búsqueda de la calidad constituyen las bases para alcanzar dichas aptitudes. Con todo, se constata, como premisa esencial impuesta por la sociedad de la información, la obligación de que los Estados realicen un urgente esfuerzo en la mejora del acceso a la formación que debe desarrollarse a lo largo del ciclo vital.

Desde una óptica más concreta, se establecen cinco objetivos o medidas prácticas para aplicar en el ámbito europeo: Fomentar la adquisición de nuevos conocimientos, acercar la escuela a la empresa, luchar contra la exclusión, dominar tres lenguas y tratar en un plano igual a la inversión en equipamiento y a la inversión en formación.

Las propuestas mencionadas suponen cierto avance significativo hacia posturas más humanistas, alejadas de planteamientos esencialmente utilitarios que otorgan a la educación la misión de resolver en buena medida los problemas de competitividad y la crisis del empleo. Por el contrario, se reconoce que la función primordial reside en ayudar a cada individuo a desarrollar todo su potencial y a convertirse en un ser humano completo y no en una herramienta para la economía. Ahora bien, no por ello se deja de insistir en que, siendo la educación y la formación los principales vectores de identificación, pertenencia y promoción social, también desempeñan un cometido esencial en materia de empleo. La novedad estriba en que, frente a planteamientos anteriores, se trata no solamente de lograr la expansión, sino también el desarrollo integral de la persona.

Al mismo tiempo, se constata que se mantiene la importancia otorgada a metas y presupuestos que defiende la educación permanente, tales como la perspectiva integral del proceso educativo en el que se incluyen los aprendizajes no formales, la función esencial de la institución escolar para fomentar el deseo y necesidad de aprender durante toda la vida, la defensa de poder ejercer el derecho a la educación en cualquier etapa de la existencia humana y la necesidad de conseguir una mayor y real igualdad de oportunidades.

\section{POLÍTICA A FAVOR DE LA EDUCACIÓN A LO LARGO DE LA VIDA}

Las consideraciones ofrecidas hasta el momento constituyen, en nuestra opinión, las variables más significativas que han influido en el diseño de los 
elementos que configuran la política actual a favor de la educación a lo largo de la vida. Con todo, dos hechos importantes van a conformar de manera definitiva los objetivos, ámbitos y cometidos que se asignan a este campo y que justifican la primacía que en los últimos años viene otorgándosele dentro de la Unión. Nos referimos, en primer lugar, a la Decisión tomada el 23 de octubre de 1995 por el Consejo y el Parlamento europeos de declarar a 1996 Año Europeo de la Educación y Formación Permanentes y a las Conclusiones del Consejo de 20 diciembre del mismo año relativas al establecimiento de los criterios fundamentales de una política activa en dicho ámbito (Diario Oficial de las Comunidades Europeas, del diez de enero de 1997).

En ambas iniciativas la educación a lo largo de la vida aparece como un concepto en evolución que abarca tanto los procesos educativos formales como no formales, y que constituye la piedra angular para afrontar con garantías de éxito los nuevos retos que requieren la participación de todos para mejorar la innovación en los sectores de la vida social y económica.

\section{UN RETO EMERGENTE: ARMONIZAR LA DIMENSIÓN SOCIOECONÓMICA Y LA CULTURAL}

Dentro del marco de las acciones de seguimiento de los Libros Blancos anteriormente citados y de la preparación de los europeos para afrontar con éxito los retos del siglo XXI, por primera vez se dedica un año europeo a la educación y formación, no siendo esta elección fortuita. Por una parte, la aceleración de las innovaciones científicas y tecnológicas exigen una adaptación cada vez más rápida y sistemática de los conocimientos y de las competencias y, por otra, los progresos realizados en los métodos de adquisición del saber, sobre todo gracias a las nuevas tecnologías, pueden y deben contribuir a la realización personal del individuo, siempre que el progreso se ponga a disposición del mayor número posible de personas.

La decisión adoptada pretendió suscitar en todo el territorio de la Unión y de los países del Espacio Económico Europeo una profunda reflexión a fin de sensibilizar a todas las personas de la necesidad de formarse durante toda su trayectoria vital. En este contexto, se pretendía iniciar un amplio debate a escala europea, nacional, regional y local sobre las condiciones de una política que hiciese posible la aplicación de dicha noción. Al mismo tiempo, se trataba de fomentar el desarrollo personal y el sentido de la iniciativa de las personas, su integración en la vida activa y en la sociedad, su participación en el proceso de decisión democrática y su capacidad para adaptarse al cambio económico, tecnológico y social.

Una de las aportaciones de mayor interés reside en que se clarifican los diferentes aspectos del ámbito objeto de estudio, entre los que figura el cometido esencial desempeñado por la educación general de alta calidad que debe 
incluir la capacidad de aprender de forma autónoma, así como la sensibilización de los padres, docentes, estudiantes, interlocutores sociales y de toda la ciudadanía, en general, sobre la conveniencia de participar en nuevas posibilidades educativas y de tomar conciencia de que el proceso educativo es una tarea inacabada. Así mismo, se subraya la necesidad de:

- Introducir sus principios en la educación inicial. Esto implica esencialmente estimular la curiosidad intelectual de los estudiantes e inducirles a aprender, aspectos que, al tiempo, constituyen los retos a los que profesores y formadores deben dar respuesta.

- Concebir de otro modo el aprendizaje, la enseñanza y la formación, puesto que la educación a lo largo de la vida es indispensable para apoyar el perfeccionamiento personal y profesional de forma continua.

- Desarrollar puentes entre la educación, la formación y el trabajo, al objeto de poder realizar una oferta formativa adaptada a públicos y demandas cada vez más diversificados.

- Fomentar el aprendizaje en las organizaciones, puesto que las empresas como "entidades autoformativas" constituyen instrumentos de cualificación nada despreciables.

La proclamación efectuada queda justificada por la necesidad de conjugar, a fin de lograr el desarrollo personal y el progreso de los pueblos, una doble dimensión: La socioeconómica y la cultural. En este sentido, cabe anotar que la fase de mutación profunda en la que ha entrado el mundo obliga a realizar un esfuerzo de adaptación considerable. Los focos tradicionales de transmisión del saber, como la escuela y el lugar de trabajo, encuentran dificultades crecientes para dar respuesta a una demanda educativa cada vez más urgente y variada. Todo ello puede ampliar los riesgos de desigualdades y de exclusión, así como fragilizar la estructura de empleo, razones que justifican el impulsar y poner en práctica la educación permanente para encontrar las soluciones adecuadas. Así mismo, con el progreso tecnológico, el individuo descubre nuevas perspectivas educativas. La responsabilización de la personas es aún más necesaria, ya que la división tradicional de la vida en un periodo de educación, otro de trabajo y otro de jubilación ha quedado obsoleta frente a una perspectiva de renovación constante de competencias. Al objeto de garantizar el perfeccionamiento personal y poner en marcha la adaptación necesaria, cada persona ha de estar motivada para adoptar iniciativas en la adquisición del saber.

\section{PRINCIPALES LÍNEAS DE ACCIÓN}

La plasmación efectiva de la idea de un proceso de enseñanza-aprendizaje inacabado en una realidad viva para los ciudadanos europeos constituye un 
nuevo hito en el desarrollo de la educación y la formación en la Unión Europea. Para avanzar en dicho sentido es necesario fomentar la motivación para integrarse en procesos continuos e impulsar una serie de líneas de actuación que procuren su desarrollo. En este sentido, los fundamentos de esta política están basados en un conjunto de principios y objetivos que muestran las coordenadas que enmarcan esta noción desde la perspectiva comunitaria. Sobre este particular, se puede constatar que cesa, en cierta medida, el enfoque fundamentalmente neo-liberal primado en determinadas propuestas anteriores, para inclinarse a favor de una orientación más humanistas, dado que entre los presupuestos básicos figuran: Contribuir al bienestar individual, cultural, social y económico, mejorar la calidad de vida de todos los ciudadanos y fomentar la igualdad de oportunidades en cada etapa dentro de la continuidad del proceso educativo por medio de la eliminación de toda forma de marginación social e impulsando la participación activa en la sociedad democrática.

Estos tres importantes objetivos constituyen los puntos de referencia básicos sobre los que se asientan los principios atribuidos a este campo, entre los que destacamos los siguientes:

- Lograr un equilibrio adecuado entre las dimensiones socioculturales y laborales. En este sentido las actividades de educación permanente deberán conjugar el desarrollo personal, cultural, cívico y social y las preocupaciones económicas y laborales e incluir, además, los principios democráticos y los valores relacionados con los derechos humanos.

- Concebir el proceso educativo de forma global, por tanto, cada fase de la educación deberá contribuir a la continuación del proceso de la educación a lo largo de la vida.

- Ampliar y diversificar la oferta educativa al objeto de brindar a todas las personas oportunidades de aprendizaje para mejorar su educación en función de sus intereses y necesidades sociales, culturales y económicas.

- Promover el desarrollo de enfoques educativos flexibles e innovadores. Estos enfoques han de fomentar el sentido de la búsqueda, la iniciativa y la motivación en los individuos durante el proceso de aprendizaje.

- Impulsar la educación y formación iniciales. La educación básica, en tanto fundamento que posibilita la adquisición de saberes y destrezas posteriores, deberá incluir, además de las nociones básicas de lectoescritura y cálculo, una importante base de conocimientos, aptitudes, actitudes y experiencias que cimienten el aprendizaje permanente.

Por otro lado, la naturaleza global del campo que nos ocupa precisa la concurrencia de diversas instituciones y contextos, por lo tanto, entre los ámbitos directamente comprometidos en la tarea de impulsar su desarrollo se encuentran: 
- El sistema educativo. Sus principales retos se centran en extender la educación infantil, especialmente para la población más desfavorecida, erradicar el analfabetismo funcional, mejorar la enseñanza de lenguas. Es imprescindible, igualmente, prestar especial atención a los jóvenes que interrumpen prematuramente sus estudios con un bajo nivel de cualificaciones, lo que provoca serias dificultades para obtener un empleo con lo que se ven excluidos de una participación activa en la sociedad. Por tanto, habrá que fomentar la colaboración entre los centros educativos y los espacios laborales a fin de crear nuevas opciones y pasarelas y ampliar la oferta de nuevas oportunidades de aprendizaje.

- Las comunidades locales. Desde el enfoque claramente integral de la educación a lo largo de la vida, las comunidades locales deben asumir mayores responsabilidades formativas, para llegar a convertirse en "comunidades educativas" donde se fomente la participación activa de todos los ciudadanos en el proceso político y democrático. Ha de analizarse el concepto de escuela como recurso de la comunidad local, y de la comunidad local como importante recurso educativo, creándose, al tiempo, vínculos entre la educación no formal que se efectúa en las comunidades locales y la educación formal.

- Las empresas. El incremento y diversificación en la oferta formativa que encierra el concepto que nos ocupa implican mejorar la colaboración entre todas las empresas vinculadas a la formación y los Gobiernos, con el fin de establecer las prioridades formativas. Al mismo tiempo, ha de fomentarse la inversión en materia de educación y formación y otorgar un mayor protagonismo a los centros de enseñanza superior para contribuir a la educación permanente e impulsar programas de educación general para adultos, con el fin de propiciar el desarrollo personal, la participación en la sociedad y la posibilidad de acceder a un empleo.

Otros componentes esenciales son la capacitación del profesorado y las estrategias dirigidas a hacer factible el derecho de todo ciudadano a educarse a lo largo de su vida. Respecto al profesorado, su motivación y capacidad de adaptación a los cambios constituyen elementos básicos, por tanto resulta fundamental garantizar una formación inicial de calidad y las posibilidades de su perfeccionamiento continuo, así como reconocer el cometido específico del educador de personas adultas. Teniendo en cuenta que son necesarias estructuras más flexibles y fluidas y más centradas en el estudiante, como estrategias principales cabe contemplar el crear mejores vías de acceso a la formación y a la vida académica, a todos los niveles, así como establecer los mecanismos adecuados y aceptables para acreditar la experiencia y nivel de aprendizaje logrado, con independencia del contexto en que se haya realizado. Las nuevas tecnologías, dado que aumentan sensiblemente el número de opciones disponibles para seguir procesos de enseñanzaaprendizaje, son especialmente adecuadas para apoyar, facilitar y estimular los procesos de educación permanente. 


\section{NUEVOS HORIZONTES}

Conviene apuntar que la expresión objeto de estudio se consagra definitivamente con las recientes Decisiones del Parlamento Europeo y del Consejo que establecen la segunda fase de los programas de acción comunitaria Socrates y Leonardo da Vinci. En la respectiva normativa, además se determina que estas iniciativas se destinan a contribuir a una Europa del Conocimiento a través de la promoción de la educación a lo largo de la vida. La citada noción queda definida como las oportunidades de educación y formación que se ofrecen a las personas a lo largo de toda su existencia para darles la posibilidad de adquirir, actualizar y adaptar de forma permanente sus conocimientos, aptitudes y competencias en el marco de la enseñanza formal o no formal, a través del aprendizaje autónomo, aumentando con ello su capacidad de inserción profesional e incrementando su aptitud para progresar en la formación y desempeñar un papel activo en la sociedad.

Ciertamente, la definición anterior presenta un marcado carácter operativo y, hasta cierto punto, ecléctico, que queda alejado de propuestas más globales y precisas, como la contenida en el conocido Informe de la UNESCO que lleva por título La educación encierra un tesoro (1997). En dicho documento se indica que la educación a lo largo de la vida abarca, desde la infancia hasta el final de la existencia, todos los medios que permiten a una persona adquirir un conocimiento dinámico del mundo, de los demás y de sí misma, combinando con flexibilidad los cuatro pilares fundamentales: Aprender a hacer, a conocer, aprender a vivir juntos y aprender a ser. A juicio de los autores, esta noción representa el requisito para un dominio de los ritmos y tiempos del ser humano, que supera con mucho la necesidad de adaptarse a los imperativos del mundo del trabajo (Delors, 1997, p. 111 y ss.). Es posible que la idiosincrasia propia de las instancias comunitarias, al igual que las diferentes circunstancias socioculturales de los países que integran la Unión, incidan en que se haya optado por una perspectiva más funcional y global que la ofrecida por la citada organización internacional.

Dentro del marco específico de la Comunidad, y con el propósito de lograr esa Europa del Conocimiento que necesitamos para abordar el siglo XXI, a la que se aludía anteriormente, consideramos que la educación a lo largo de la vida debe asumir, al menos, dos retos importantes: Promover las capacidades y destrezas que demanda la sociedad cognitiva y contribuir al desarrollo de una ciudadanía activa. Si bien es cierto que el sistema educativo ha de responsabilizarse, en primer término, de estas finalidades, no lo es menos que las mismas pueden y deben afrontarse también desde procesos de aprendizaje no formales e informales y en distintos espacios y tiempos del ciclo vital.

La sociedad del conocimiento obliga a que todas las personas adquieran, completen y perfeccionen, en cualquier etapa de su vida, entre otras, las siguientes capacidades y destrezas: Encontrar información en unos circuitos 
múltiples y complejos que ya se transmite y se transforma a gran velocidad. Por tanto, no solamente es necesario conocer los medios tradicionales, sino que hay que aprender adecuadamente a utilizar los distintos soportes que van surgiendo gracias a las nuevas tecnologías. Junto a este objetivo se ha de adquirir la habilidad de seleccionar la información adecuada, puesto que el problema actual no es la falta de información, sino, más bien, cómo distinguir la que resulta verdaderamente significativa para los propósitos perseguidos en cada momento. Es preciso, por tanto, disponer de los conocimientos básicos que faciliten la identificación de aquellos datos que resultan esenciales para responder a las cuestiones planteadas. Ahora bien, siendo imprescindibles los dos aspectos anteriores, quedarían sin contenido si no se orientasen a transformar la información en conocimiento. Aunque la construcción del conocimiento implica disponer de la información apropiada, es necesario realizar procesos de interpretación que conduzcan a un aprendizaje activo y que la conviertan en nuevos saberes.

Por otra parte, se va vislumbrando una figura de Europa no sólo de la competencia y del mercado, sino una Europa de los ciudadanos, que demanda conciliar la identidad y pluralidad de culturas para construir una sociedad multicultural, una ciudadanía supranacional. A pesar de los requerimientos efectuados y de las acciones emprendidas, estamos aún muy lejos de lograr un nivel aceptable al respecto. Conseguir esta nueva Europa es responsabilidad de todos. En este sentido, en primer término, y a fin de tomar conciencia de nuestra pertenencia a una comunidad supranacional, debemos aprender a compartir valores espirituales, morales y cívicos que no hay que inventar, pues forman parte del acervo cultural común y que ahora, por influjo del materialismo, del relativismo y del utilitarismo, llevan ya tiempo en decadencia. No ha de olvidarse, como indica López-Barajas (2000, p. 12), que la civilización cristiana, el mundo clásico griego, la romanización, vertebradas por la Ilustración moderna han influido significativamente en el pensamiento europeo actual.

Fomentar el respeto y ejercicio de los derechos humanos, los principios democráticos, la tolerancia, la justicia social, la participación y la solidaridad, así como el total rechazo a toda expresión de racismo y xenofobia y de exclusión, requieren tanto o más acción que enseñanza formal, y estas acciones deben ser compartidas por todos y desde una proyección tanto inicial como continua. La educación para la ciudadanía es un sólido reto para la educación a lo largo de la vida, pero no sólo en el sentido de descubrimiento o incorporación de nuevos elementos o contenidos de aprendizaje, sino también en cuanto requiere una conformación nueva de las sensibilidades y mentalidades educativas.

A título de síntesis final, cabe señalar que se ha producido en el marco de la Unión un creciente interés, como se ha podido comprobar, por la educación permanente, noción donde se inscriben y toman auténtico sentido las iniciativas orientadas a propiciar la educación de personas adultas y la for- 
mación profesional continua de los trabajadores. La evolución en este sentido ha sido verdaderamente importante, pues si una de las primeras inquietudes en materia educativa de la Unión ha sido la formación profesional, en nuestros días el centro de atención recae en la educación a lo largo de la vida, principio educativo que día a día va retomando la tradición humanista y democrática que la debe caracterizar.

Dentro de este contexto, como profesionales de la educación y como ciudadanos europeos, se nos presenta un desafío importante: Contribuir a la construcción del futuro de la Europa de la paz, del bienestar común, solidario y compartido, desafío en el que la educación a lo largo de la vida está llamada a desempeñar una función primordial.

\section{BIBLIOGRAFÍA}

BÎRCEA, C. (1996). Education for democratic citizenship. Strasbourg: Council of Europe.

Centro para la Investigación e Innovación en la Enseñanza de la OCDE (1997). Análisis del Panorama Educativo. París: OCDE.

Comisión Europea (1995). La cooperación en educación en la Unión Europea. Luxemburgo: Educación, Formación y Juventud, Oficina de Publicaciones Oficiales de la Comunidades Europeas.

Comisión de las Comunidades Europeas (1987). Tratados constitutivos de las Comunidades Europeas. Luxemburgo: Oficina de Publicaciones Oficiales de las Comunidades Europeas.

- (1993). Libro Verde sobre la Dimensión Europea de la Educación. Luxemburgo: Oficina de Publicaciones Oficiales de las Comunidades Europeas

- (1994). Libro Blanco. Crecimiento, Competitividad, Empleo. Retos y pistas para entrar en el siglo XXI. Luxemburgo: Oficina de Publicaciones Oficiales de las Comunidades Europeas.

- (1995). Libro Blanco sobre la educación y la formación. Enseñar y Aprender. Hacia la sociedad cognitiva.
Luxemburgo: Oficina de Publicaciones Oficiales de las Comunidades Europeas.

- (1997). Por una Europa del Conocimiento. Luxemburgo: Oficina de Publicaciones Oficiales de las Comunidades Europeas.

- (1999). El Tratado de Amsterdam. Luxemburgo: Oficina de Publicaciones Oficiales de las Comunidades Europeas.

- Conclusiones del Consejo, de 20 de diciembre de 1996, sobre una politica de educación permanente. Bruselas: (97/C 7/02). Diario Oficial de las Comunidades Europeas del 10 de enero de 1997

CRESSON, E. (1996). La educación y la formación permanentes: un reto para cada ciudadano. Revista Española de Pedagogía, año LIV, 204, pp. 223-229.

Decisión $n^{\circ}$ 2493/95/CE del Parlamento Europeo y del Consejo, de 23 de octubre de 1995, por la que se declara 1996, como el "Año Europeo de la Educación $y$ de la Formación Permanentes". Diario Oficial n" L 256 de 26/10/1995 P. $0045-0048$.

Decisión del Consejo (1999/382/CE), de 26 de abril de 1999, por la que se establece la segunda fase del programa de acción 
comunitario en materia de formación profesional Leonardo da Vinci. Diario Oficial de las Comunidades Europeas n". L 146/33 de 11/6/1999.

Decisión $n^{\circ}$ 253/2000/CE del Parlamento Europeo y del Consejo de 24 de enero de 2000 por la que se establece la segunda fase del programa de acción comunitario en materia de educación Sócrates. Diario Oficial $n^{\circ}$ I. $28 / 1$ de 03/02/2000.

DELORS, J. (Coord.) (1997). La educación encierra un tesoro. Informe a la UNESCO de la Comisión Internacional sobre la educación para el siglo $X X I$, presidida por Jacques Delors. Madrid: Santillana.

ETXEBERRÍA, F. (1996). Política educativa de la Unión Europea: evolución reciente y perspectivas de futuro. Revista Española de Pedagogía, año LIV, 204, pp. 231-247.

GARCÍA GARRIDO, J.L. (1995). Educación y unidad europea. Madrid: Colegio de Doctores y Licenciados de Madrid. Universidad de Otoño. Documento policopiado.

GARCÍA MÍNGUEZ, J. (1998). La cara oculta de las políticas sociales europeas. En J. García Mínguez y A. Sánchez Sánchez (Coords.), Políticas Sociales y Educación Social. Actas del XIII Seminario de Pedagogía Social (pp. 57-80). Granada: Grupo Editorial Universitario.

LÓPEZ-BARAJAS ZAYAS, E. (2000). La elaboración del pensamiento europeo. En E. López Barajas Zayas (Coord.), La Educación y la construcción de la Unión Europea. Madrid: UNED.

MARÍN IBÁÑEZ, R. (1992). Política de formación y educación de la Comunidad Económica Europea. En Cuestiones actuales sobre educación (pp. 365384). Madrid: UNED.

MEDINA RUBIO, R. (1997). El marco competencial de la educación y de la cliltura en el Tratado de la Unión Europea. En M.A. Santos Rego, Política educativa en la Unión Europea después de Maastrich (pp.71-91). Santiago de
Compostela: Escuela Galega de Administración Pública, Xunta de Galicia.

- (1999). La política educativa comunitaria y la construcción de una nueva "polis" europea. Congreso Nacional de Teoría de la Educación, Oviedo. Documento policopiado.

MOLERO PINTADO, A. (1999). Política y educación en la Unión Europea: un proyecto emergente. Revista de Ciencias de la Educación, 178-179, pp. 243-259.

REQUEJO OSORIO, A. (1998). Sociedad del aprendizaje y tercera edad. Revista Interuniversitaria Teoría de la Educación, vol. 10, pp. 145-167.

RODRÍGUEZ, V.M. (1993). De Roma a Maastricht: 35 años de cooperación comunitaria en educación. Revista de Educación, 301, pp. 7-24.

SANZ FERNÁNDEZ, F. (1995). La educación de personas adultas en los países de la Unión Europea. En F. Sanz. Fernández (Dir.), La formación en educación de personas adultas (249298). Madrid: UNED-MEC.

SARRATE CAPDEVILA, $\mathbf{M}^{\mathrm{a}}$. L. (2000). Conciencia europea y educación de adultos. En E. López-Barajas Zayas (Coord.), La Educación y la construcción de la Unión Europea. Madrid: UNED.

Table rond des Industriels Européens (1994). Une éducation européenne: vers une société qui apprend. Bruxelles: Table Ronde des Industriels Européens.

TEDESCO, J.C. (1998). Los fenómenos de segregación y exclusión social en la sociedad del conocimiento y de la información. II Congreso Estatal de Educación Social. Documento policopiado.

- (1998). CONFITEA. V Conferencia Intemacional de Educación de las Personas Adultas. Declaración de Hamburgo y La Agenda para el futuro. Hamburgo: Instituto de la UNESCO para la Educación.

VV.AA. (1997). Los principios fundadores de la ciudadanía europea. Actas de la Primera Convención Universitaria Europea. Barcelona: Dabar. 


\section{RESUMEN}

Analizar la evolución de las políticas comunitarias en materia educativa constituye un método especialmente adecuado para comprender la percepción de la Unión Europea respecto de sus intereses y objetivos sociales y culturales en su marco geográfico. El impulso otorgado a las modernas concepciones educativas, desde la mera contemplación inicial de la formación profesional hasta la actual orientación hacia una educación a lo largo de la vida de los ciudadanos, representa el reconocimiento definitivo de los programas que abren los nuevos horizontes formativos en la moderna sociedad del conocimiento.

En el presente artículo se revisan las cuestiones mencionadas, explicando los nuevos paradigmas, politicas e iniciativas educativas en la nueva Unión Europea, encaminados a desarrollar una educación durante todo el ciclo vital.

Palabras clave: Políticas comunitarias, formación profesional, educación a lo largo de toda la vida, sociedad del conocimiento, iniciativas educativas.

\section{ABSTRACT}

Analysing the evolution of the communitary policies in education, constitutes a proper method in order to understand European Union's perception of social and cultural interest and targets in its geographic scope. The impulse of the modern educative conceptions, from the mere initial contemplation to today's orientation to lifelong education, represents the definitive recognition of the programs that open new educational horizons in nowadays society.

In this article, we review all this issues, explaining the new educative paradigms, policies and initiatives in the European Union, set to develop an education for all the vital cycle.

Key words: Communitary policies, lifelong education, educative initiatives, knowledge society. 